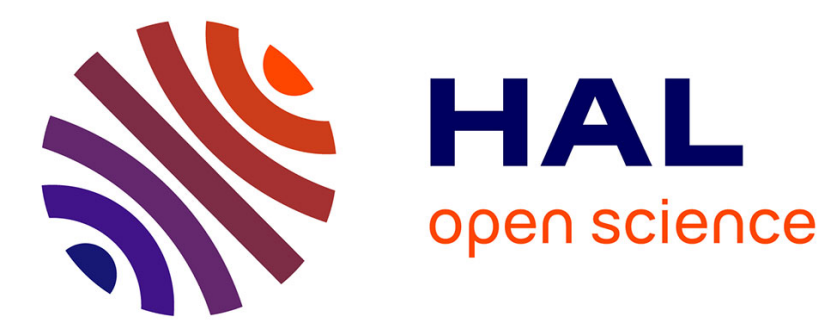

\title{
L'approche à la saturation de la magnétostriction
}

Louis Neel

\section{To cite this version:}

Louis Neel. L'approche à la saturation de la magnétostriction. J. Phys. Radium, 1954, 15 (5), pp.376-378. 10.1051/jphysrad:01954001505037601 . jpa-00234937

\section{HAL Id: jpa-00234937 https://hal.science/jpa-00234937}

Submitted on 1 Jan 1954

HAL is a multi-disciplinary open access archive for the deposit and dissemination of scientific research documents, whether they are published or not. The documents may come from teaching and research institutions in France or abroad, or from public or private research centers.
L'archive ouverte pluridisciplinaire HAL, est destinée au dépôt et à la diffusion de documents scientifiques de niveau recherche, publiés ou non, émanant des établissements d'enseignement et de recherche français ou étrangers, des laboratoires publics ou privés. 
liquide intersticiel est joué par l'air lui-même. Ces filtres sont donc transparents dans les régions du spectre où l'indice de réfraction de la matière dispersée dans l'air est voisin de $\mathrm{I}$. On résumera leur principe en remarquant que les radiations de ces domaines se propagent en ligne droite dans le filtre, sans réflexion ni réfraction. Les pertes s'y réduisent donc à l'absorption de la matière. Les autres radiations, au contraire, sont réfractées et réfléchies dans toutes les directions pourvu que leur longueur d'onde soit inférieure à la dimension des particules. Deux substances se présentent immédiatement pour la région $30-43 \mu$ : $\mathrm{NaCl}$ et $\mathrm{KCl}$. Leurs indices deviennent égaux à I vers 32 et $37 \mu$ et restent assez longtemps voisins de $\mathrm{I}$, n'atteignant $\mathrm{I}, 2$ qu'à 27 et $30 \mu$ respectivement.

Cette solution, préconisée par Barnes en I936 [3], n'avait jamais été utilisée et, dans la littérature récente, seul J. Lecomte rappelle ses possibilités [4]. Pourtant l'introduction de matières plastiques transparentes dans l'infrarouge lointain, devait faciliter la préparation de ces filtres en fournissant un support aux grains qui doivent disperser par réfraction les radiations parasites. Nous avons utilisé des films très minces en polyéthylène, transmettant plus de 85 pour roo vers 3 o $\mu$. Suivant la grosseur des particules cristallines et le nombre de couches que l'on dépose, on réalise des filtres ayant soit les caractéristiques décrites par Barnes : bande passante étroite, mais transmission assez faible (4o à 50 pour ıoo au maximum), soit au contraire bande large et transmission élevée.

Ce sont ces derniers qui conviennent le mieux pour un spectrographe à réseau. Ils laissent encore passer un peu de lumière parasite, mais un même filtre peut être employé dans un domaine s'étendant sur plus de ro $\mu$. Ils sont d'autant plus pratiques dans ce cas que l'on peut utiliser comme support, les parois mêmes de la cuve interposée sur le trajet lumineux. La transmission n'est pas diminuée par des réflexions sur de nouvelles feuilles de polythène et deux filtres ainsi séparés sont plus efficaces qu'un seul d'épaisseur double.

Nous avons ainsi préparé un filtre double au $\mathrm{KCl}$ qui fait perdre moins de 35 pour roo de l'énergie contenue dans le spectre du premier ordre entre $3 o$ et $43 \mu$ et qui élimine, dans ce domaine, 7o pour roo environ de la lumière parasite apportée par les spectres d'ordre supérieur.

Nous l'avons utilisé pour l'étude de la structure fine de l'absorption de l'alcool méthylique [2] et des méthylamines entre $3 o$ et $43 \mu$. Pour celles-ci, les maxima d'absorption semblent situés à 336, 233,6 et $269,5 \mathrm{~cm}^{-1}$, respectivement pour les tri-, di- et mono-méthylamines. Cette dernière fréquence est vraisemblablement due à la vibration de torsion du groupement $\mathrm{CH}_{3}$ par rapport au groupement $\mathrm{NH}_{2}$. Plyler, après avoir étudié le spectre de $\mathrm{I}$ à $20 \mu$ seulement, pensait pouvoir la situer à $620 \mathrm{~cm}^{-1}$ [5]. Nous espérons raccorder ces deux études en prolongeant notre spectre de 30 à $20 \mu$. Nous pourrons alors interpréter l'ensemble des bandes avec plus de certitude.
[1] Oetuen R. A. - J. Opt. Soc. Amer., 1952, 42, 559 .

[2] Hadni A. - C. R. Acad. Sc., г $953,236,1761$; 1954 , 238, 573 .

[3] Barnes R. B. et Bonner L. G. - Phys. Rev., i936, 49, 732 .

[4] Lecomte J. - Le rayonnement infrarouge. GauthierVillars, Paris, 1948.

[5] Cleaves A. P. et Plyler E. K. - J. Chem. Phys., I $939,7,563$.

\section{L'APPROGHE A LA SATURATION DE LA MAGNÉTOSTRICTION}

Par M. Louis NÉEL.

La loi d'approche à la saturation de la magnétostriction longitudinale $\lambda$ d'un corps ferromagnétique cubique polycristallin a été étudiée par Schlechtweg [1] qui a montré que le premier terme du développement en série était un terme en $\frac{\mathrm{I}}{\boldsymbol{H}}$. Récemment, Lee a calculé [2] le terme suivant en $\frac{\mathrm{I}}{\mathrm{H}^{2}}$, terme qui subsiste seul dans le cas d'une magnétostriction isotrope. En prenant comme point de départ l'existence d'une énergie magnétocristalline $F_{m}$ donnée par

$$
F_{m}=K\left(\alpha_{1}^{2} \alpha_{2}^{2}+\alpha_{2}^{2} \alpha_{3}^{2}+\alpha_{3}^{2} \alpha_{1}^{2}\right)
$$

et suivant la voie indiquée par Becker [3] dans l'étude de 'l'approche à la saturation de l'aimantation, Lee montre que la loi d'approche à la saturation de la magnétostriction longitudinale d'un monocristal aimanté suivant une direction de cosinus directeurs $\alpha_{1}, \alpha_{2}, \alpha_{3}$ (rapportés aux axes quaternaires choisis comme axes de coordonnées) s'écrit

$$
\begin{aligned}
\lambda=\frac{3}{2}\left(\lambda_{100}-\lambda_{111}\right)\left[S_{4}\right. & -\frac{\mathrm{I}}{3}+2 \rho\left(S_{6}-S_{4}^{2}\right) \\
& \left.+\rho^{2}\left(7 S_{8}-17 S_{4} S_{6}+10 S_{4}^{3}\right)\right] \\
& +\lambda_{111}\left[\mathrm{I}-\frac{3}{2} \rho^{2}\left(S_{6}-S_{4}^{2}\right)\right] .
\end{aligned}
$$

Dans cette formule, on a posé

$$
p=\frac{K}{H J}, \quad S_{n}=\alpha_{1}^{n}+\alpha_{2}^{n}+\alpha_{3}^{n}
$$

tandis que $\lambda_{100}$ et $\lambda_{111}$ désignent la magnétostriction à saturation suivant les axes quaternaire et ternaire; $J$ est l'aimantation spontanée, $H$ le champ magnétique. En faisant la moyenne pour toutes les directions de l'espace, il trouve ensuite, pour un ensemble de cristallites orientés au hasard, la loi d'approche suivante :

$$
\begin{aligned}
\lambda= & \frac{9 \lambda_{100}+3 \lambda_{111}}{5} \\
& +\left(\lambda_{100}-\lambda_{111}\right)\left[\frac{8}{5 \cdot 7} p-\frac{32}{7 \cdot 1 \mathrm{I} \cdot \mathrm{I} 3} p^{2}\right] \\
& \quad-\lambda_{111} \frac{8}{5 \cdot 7} \rho^{2}+\ldots
\end{aligned}
$$

Cette expression (2) ne s'applique qu'à des cris- 
tallites indépendants. On sait d'ailleurs [4], [5] que dans le cas de l'approche à la saturation de l'aimantation, on tient compte des interactions entre les cristallites en multipliant le terme en $\frac{\mathrm{I}}{\boldsymbol{H}^{2}}$ de la loi d'approche relative aux cristallites indépendants par un certain coefficient $\frac{G}{2}$, plus petit que l'unité. Pour tenir compte des interactions, Lee propose donc de remplacer $H$ dans l'expression (2) par un champ interne $H^{\prime}$ relié au champ appliqué $H$ par la relation

$$
\left(\frac{H}{H^{\prime}}\right)^{2}=\frac{G}{2} \text {. }
$$

Il me semble préférable d'effectuer la correction d'interactions en multipliant par $\frac{G}{2}$ l'ensemble des termes en $p$ et en $p^{2}$ de l'équation (2). Cette manière de procéder peut se justifier par l'examen de la méthode [5] que nous avons utilisée autrefois pour résoudre le problème des interactions. Au voisinage de la saturation, un ensemble de $N$ moments atomiques possède ${ }_{2} \mathrm{~N}$ degrés de liberté d'oscillation. Par une combinaison linéaire convenable de ces degrés de liberté, on peut obtenir un système équivalent comprenant aussi $2 N$ termes, mais qui peuvent se séparer en deux groupes de $N$ termes chacun, possédant les propriétés suivantes. Dans le premier groupe, la divergence du vecteur représentant les variations de l'aimantation est nulle : les oscillations ne font donc pas apparaître de champs de dispersion et peuvent ainsi se produire librement. Dans le second groupe, au contraire, la divergence n'est pas nulle de sorte que les oscillations des moments atomiques donnent naissance à un champ magnétique, avec une grande augmentation de l'énergie potentielle : pratiquement, l'amplitude de telles oscillations ne peut être que faible. Dans un large domaine de champs, tout se passe donc comme si la moitié des degrés de liberté était bloquée : la présence des interactions divise donc par deux les écarts à la saturation. Effectivement, le calcul montre [5] que, dans le domaine de champs qui nous intéresse, $\frac{G}{2}$ est toujours voisin de $\frac{\mathrm{I}}{2}$. Évidemment, ce raisonnement doit s'appliquer aussi bien à la magnétostriction qu'à l'aimantation. Pratiquement, la correction nécessaire s'obtiendra ainsi en première approximation en multipliant par l'ensemble des termes en o et en $c^{2}$ de l'équation (2).

Nous avons comparé à l'expérience les résultats théoriques ainsi obtenus, dans le cas du fer et du nickel. Pour le fer, nous avons adopté :

$$
\begin{gathered}
K=-5.10^{5} \mathrm{ergs} / \mathrm{cm}^{3}, \quad \lambda_{100}=20 \cdot \mathrm{IO}^{-6}, \\
\lambda_{111}=-20 \cdot 10^{-6}, \quad J=\mathrm{I} \text { б00 u. é. m. }
\end{gathered}
$$

Pour le nickel, nous avons pris :

$$
\begin{gathered}
K=5, \mathrm{I} \cdot \mathrm{IO}^{6} \mathrm{ergs} / \mathrm{cm}^{3}, \quad \lambda_{100}=-54 \cdot 10^{-6}, \\
\lambda_{111}=-27 \cdot 10^{-6}, \quad J=\text { 4́ } 0 .
\end{gathered}
$$

En ce qui concerne les résultats expérimentaux, nous avons utilisé dans le cas du nickel d'excellentes mesures de Lee [6] ainsi que la représentation adoptée par lui où $\frac{\mathrm{d} \lambda}{\mathrm{d} H}$ est porté en fonction de $\frac{\mathrm{I}}{H^{3}}$. Pour le fer,

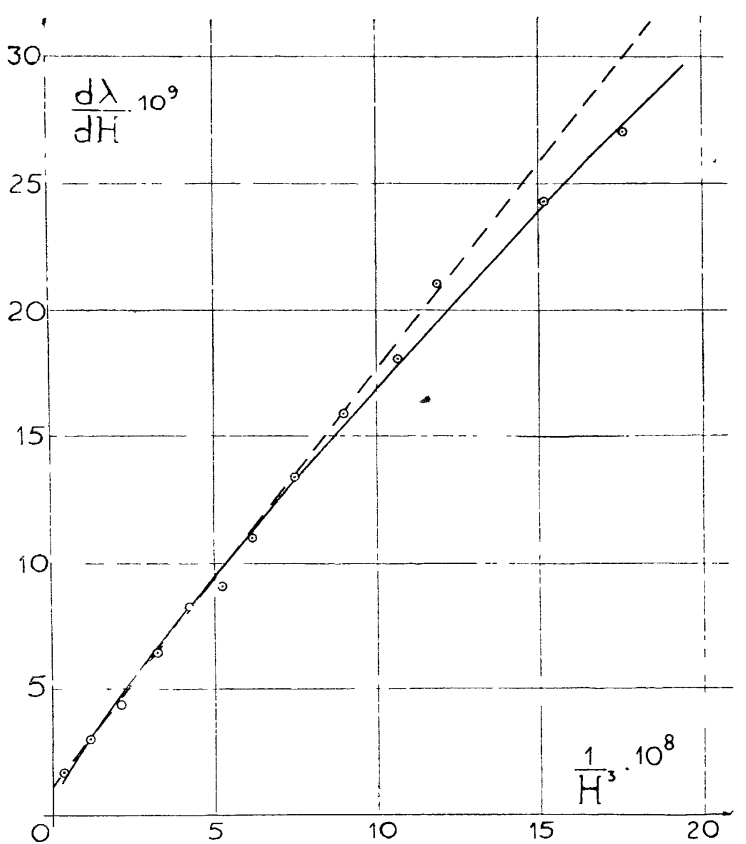

Fig. I. - Approche à la saturation de la magnétostriction longitudinale du nickel.

Les cercles correspondent aux points expérimentaux de Lee; la droite en pointillé est celle que Lee avait tracée pour représenter ses expériences. La courbe en trait plein correspond à la formule théorique du texte avec un facteur de correction égal à $\frac{r}{\text { ? }}$.

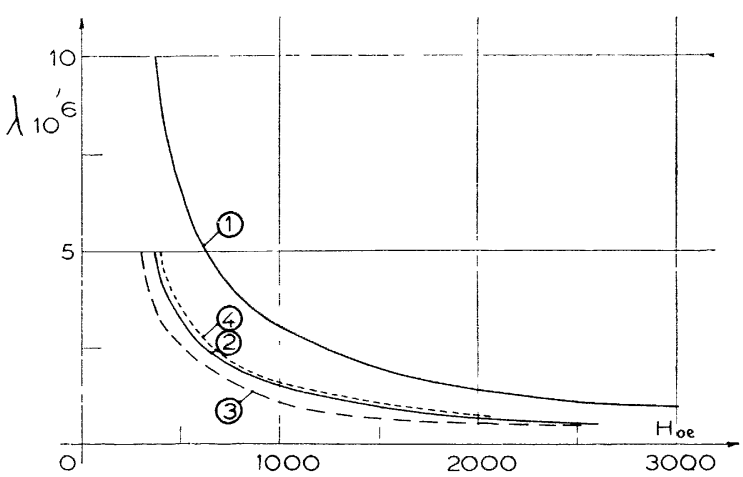

Fig. 2. - Approche à la saturation de la magnétostriction longitudinale du fer.

1. Courbe théorique sans facteur de correction d'interactions; 2. Courbe théorique avec un facteur de correction égal à $\frac{\mathrm{I}}{2} ; 3$. Résultats expérimentaux de Weil et Reichel; 4. Résultats expérimentaux de Kornetzki.

nous avons utilisé des résultats de Kornetzki [7], corrigés du champ démagnétisant $(N=0$, I 35$)$ et du terme de volume proportionnel au champ, ainsi que 
ceux de Weil et Reichel [8] obtenus à Grenoble par la méthode des jauges à résistance. Les figures $\mathrm{I}$ et 2 montrent que l'accord de la théorie avec l'expérience est satisfaisant. En particulier, dans le cas du fer, nous avons également représenté (courbe 2, fig. I) la courbe théorique obtenue sans faire la correction d'interactions : elle est beaucoup moins satisfaisante que la courbe corrigée. Ce serait encore plus frappant dans le cas du nickel.

Ces résultats font également ressortir que, dans les champs élevés, l'allure des variations de la magnétostriction s'interprète facilement par les théories existantes et ne semble pas présenter d'anomalies.

$$
\text { Manuscrit reçu le } 24 \text { février } 1954 \text {. }
$$

[1] Rüdiger O. et Schlec̀ntweg H. - Ann. Physik, r94r, 39, I et г942, 41, no 2; Techn. Mitteilungen Krupp, Forschungberichte, $194 \mathrm{1}, \mathbf{4}, \mathrm{I}$ et $1942,5,87$

[2] Lee E. W. - Communication personnelle. Proc. Phys. Soc., sous presse.

[3] Becker R. et Döring W. - Ferromagnetismus, Springer, Berlin, 1939 .

[4] Holstein T. et Primakoff H. - Phys. Rev., i94i, 59, 388.

[5] Néel L. - C. R. Acad. Sc., 1945, 220, 8 г.4; J. Physique Rad., i 948 , 9, г 93.

[6] Lee E. W. - Proc. Phys. Soc., B, I952, 65, I62.

[7] Konnetzki M. - Z. Physik, i933, 87, 56o.

[8] Weil L. et Reicher K. - Comm. Soc. franç. Physique, Grenoble, I I février ig54. J. Physique Rad., I954, 15.

\section{INDUCTION NUGLEAIRE EN GHAMP ALTERNATIF}

Par C. Manus, R. Mercier,

École Polytechnique de l'Université de Lausanne,

G. J. Béné, P. M. Denis et C. R. Extermann, Institut de Physique, Université de Genève.

Le dispositif classique d'observation directe de la résonance magnétique nucléaire comprend, outre le système radioélectrique d'émission et de réception du signal RF, un champ constant qui fixe la fréquence de Larmor et un balayage, généralement de basse fréquence et de faible amplitude.

A l'aide d'un dispositif réalisé par l'un de nous (C. M.), nous avons observé la résonance magnétique des protons sans champ constant avec un champ alternatif à $50 \mathrm{c} / \mathrm{s}$, d'amplitude suffisante pour traverser quatre fois par période la valeur de résonance correspondant à la fréquence du dispositif RF. Deux des signaux obtenus correspondent aux valeurs positives du champ magnétique et les deux autres aux valeurs négatives.

Ce résultat a nécessité :

Io L'abaissement de la fréquence de Larmor des protons pour l'amener à une valeur correspondant aux champs aisément accessibles à l'aide d'un générateur de faible puissance. Nous avons ainsi observé la résonance des protons à la fréquence de $350 \mathrm{kc} / \mathrm{s}$.

$2^{\circ}$ La construction de bobines d'Helmholtz dépourvues de toute pièce métallique pour éviter tout èffet d'induction. $3^{\circ}$ Une étude préalable des conditions de relaxation du système, la vitesse de passage et l'absence de toute polarisation nucléaire influant très fortement sur l'amplitude et la forme du signal.

Le résultat obtenu se prête remarquablement à un exposé synthétique des caractères particuliers de l'induction nucléaire et des effets de relaxation.

1. On observe en effet que, pour le même noyau, si le champ magnétique change de sens, le signal est renversé

On a ainsi directement la preuve de l'effet directionnel de la méthode de Bloch qui, à l'instar de celle de Purcell, permet de connaître le signe relatif de deux moments nucléaires dans le même champ magnétique ou de deux champs magnétiques avec le même noyau.

2. La variation du courant d'alimentation des bobines modifie considérablement de manière mesurable la vitesse de passage à la résonance. Il est ainsi facile de montrer les variations d'aspect du signal suivant les valeurs de la vitesse de passage et des temps de relaxation.

3. L'extinction ou l'apparition du signal lorsque la valeur de crête du champ alternatif est égale à la valeur de résonance est mesurable avec une précision donnée par la largeur de la raie de résonance. On a ainsi étendu l'emploi de la résonance nucléaire à la mesure et à la comparaison des champs et des courants alternatifs.

Signalons l'application possible de ce dispositif à la mesure du champ magnétique terrestre par une méthode de zéro. Le champ effectif à l'emplacement du noyau est, en effet, la résultante du champ alternatif et de la composante du champ terrestre suivant l'axe du système. Si ce dernier n'a pas été compensé exactement, les quatre signaux de résonance n'apparaîtront pas simultanément mais par groupes de deux. On observe d'abord : les signaux pour lesquels les deux champs sont parallèles, puis, pour une valeur un peu plus élevée du courant alternatif d'alimentation, les deux signaux pour lesquels les champs sont antiparallèles. La variation de courant qui sépare l'apparition des deux groupes de signaux, dont la précision est donnée par la finesse des résonances, peut être mesurée à l'aide d'un pont et l'on en déduit, en valeur absolue, la composante du champ terrestre suivant l'axe du système.

$$
\text { Manuscrit reçu le i } 7 \text { février } 1954 \text {. }
$$

\section{ÉTUDE EXPÉRIMENTALE DES ÉLECTRONS DE L'ATOME RÉSIDUEL ÉJEGTÉS DE LEURS ORBITES LORS DE LA DÉSINTÉGRATION DE ${ }^{32} \mathrm{P}$}

Par Georges Charpak et Francis Suzor, Laboratoire de Chimie nucléaire, Collège de France.

L'étude des rayonnements émis simultanément avec la particule $\beta$ lors de la désintégration $d u{ }^{32} \mathrm{P}$ a été faite avec deux compteurs $2 \pi$ accolés dont on étudiait les impulsions en coïncidence [1]. 